\title{
Synthesis and Development of $\mathrm{Gd}^{3+}-\mathrm{ALGDG}_{2}-\mathrm{C595}$ as MR Imaging Contrast Agent
}

\author{
Mehdi Mirzaei $^{{ }^{*}}$, Mohammadali Mohagheghi ${ }^{2}$, Daryoush Shahbazi-Gahrouei ${ }^{3}$ \\ ${ }^{1}$ Department of Medical Physics and Biomedical Engineering, School of Medicine, Shahid Beheshti University of Medical Sciences, \\ Tehran, Iran; ${ }^{2}$ Cancer Institute Research Center, Tehran University of Medical Sciences, Tehran, Iran; ${ }^{3}$ Department of Medical Phys- \\ ics and Medical Engineering, School of Medicine, Isfahan University of Medical Sciences, Isfahan, Iran. \\ Email: ${ }^{*}$ m_mirzaei@sbmu.ac.ir,dr_mehdimirzaei@yahoo.com
}

Received October $10^{\text {th }}, 2012$; revised November $20^{\text {th }}, 2012$; accepted December $28^{\text {th }}, 2012$

\begin{abstract}
Magnetic Resonance imaging (MR imaging) as a powerful non-invasive modality is of high global interest for early cancer detection. The aim of this study was the synthesis of nanodendrimer and its conjugate with monoclonal antibody C595 against breast cancer cell, followed by its chelating with gadolinium for its magnetic property. First, anti-MUC-1 monoclonal antibody C595 was coupled to a biodegradable biocompatible Anionic Linear Globular Dendrimer $\mathrm{G}_{2}$ (having polyethylene glycol core and citric acid shell). Then prepared nanocomplex loaded by gadolinium to make novel agent of functional MR imaging. Anticancer effects and MR imaging parameters of the prepared nanoconjugate was investigated under in vitro conditions doing performing several studies such as evaluation of monoclonal antibody C595 binding to mucine-1 (MUC-1) cell, its purification, size of nanoconjugate and relaxivity measurements. The obtained data showed a powerful relaxations as well as selective MUC-1 antigen binding to the cell. Based on the findings from the present research $\mathrm{Gd}^{3+}-\mathrm{ALGDG}_{2}-\mathrm{C} 595$ nano-probe may be a potential breast molecular imaging and therapeutic agent. However, further investigations by in vivo studies and clinical trials are in the pipeline.
\end{abstract}

Keywords: $\mathrm{Gd}^{3+}-\mathrm{ALGDG}_{2}$; Mucine-1; Monoclonal Antibody C595; MR Imaging; in Vitro

\section{Introduction}

Selective imaging is essentially an important means of targeting cancer. Better outcomes obtained by site-specific delivering of contrast agents to tumors. The fact highly depends on specific carrier for the cancer cells that can be recognized by attractive agents such as antibodies or ligands. In MR imaging of cancer, contrast agent size plays an important role in the efficacy and success of the biomedical imaging. Microsize has many obvious disadvantages comparing to nanosize in MR biomedical imaging, because of the size of cellular or subcellular units [1-4]. Conventional microsize cancer drug delivery suffers from some insufficiencies of delivery such as, inappropriate targeting, toxicological effects or impaired transport to the cancerous site [5-8]. Moreover, microsized cell entering carriers cannot traverse in a passive action through cells or pores including tumoric cells with pore sizes up to $380-780 \mathrm{~nm}$. In conclusion, the best system for tumor MR applications would be a targeted nano-carrier complex [7,9-11]. Delivering intact drugs using polymeric carriers is of global interest, dendrimers (nanosized polymers) have been explored for the

${ }^{*}$ Corresponding author. target specific delivering of hydrophobic pharmaceuticals, including anticancer drugs, and MR contrast agents. The chemico-physical characteristics of dendrimers, including their monodispersity, water solubility, drug loading ability, and large number of functionalizable peripheral groups, make these macromolecules appropriate candidates for evaluation as carrier for MR tumor imaging agents or therapeutics. This is often possible by means of safe nanocarriers such as anionic linear globular dendrimers [10-12]. Yet, not all of the dendrimers are suitable in MR biomedical imaging; biocompatible properties like lipophilicity $(\log \mathrm{P})$, biodegradability, noncytotoxicity, non immunogenicity characteristics must be checked for dendrimers under in vitro conditions. One of the verified dendrimer, one generations of a highly water soluble $\mathrm{ALGDG}_{2}$ (anionic linear globular dendrimer $\mathrm{G}_{2}$ ), MW $<2000 \mathrm{Da}$, having poly ethylene glycol core and citric acid periphery surface, was selected for the synthesis and subsequent MR agent coupling. This dendrimer has shown a good capacity of drug loading in cancer therapy, previously [12]. Monoclonal antibodies are among the best selective target specific carrier of pharmaceuticals [13]. One of the targets at breast tumor is breast specific membrane antigen (MUC-1). MUC-1 is a high mo- 
lecular weight transmembrane glycoprotein antigen [14]. Thus, tumor-associated MUC-1 is a promising molecular target for a novel imaging or therapy for breast cancer patients.

$\mathrm{C} 595$ is an $\mathrm{IgG}_{3}$, a monoclonal antibody (Mab) against human MUC-1 [12]. Several studies have shown that Mab C595 is a useful antibody in combination with other therapeutic methods to improve the therapy of the breast cancer [8].

MR imaging is a precise more sensitive and noninvasive diagnostic way based on the differences between relaxation rates of water protons and provides important graphical images. Current MR imaging contrast agents such as gadolinium $\left(\mathrm{Gd}^{3+}\right.$-diethylenetriaminepentaacetic acid (DTPA)-dimeglumine (Magnevist ${ }^{\circledR}$ ) improve tissue discrimination in the MRI images but not as well as nuclear radiopharmaceuticals which act specifically $[12$, 15]. Then, considering the MR imaging method safer than nuclear radiopharmaceutical, improving its efficacy by targeting technology could have more clinical benefits and made it superior than nuclear radiopharmacy.

This study is the first to describe synthesis and in vitro evaluations of a novel nanoconjugate contains $\mathrm{ALGDG}_{2}$ loaded $\mathrm{Gd}^{3+}$ and Mab C595 as a selective breast molecular imaging and therapeutic agent. In fact, conjugation of dendrimer (anionic linear globular) to $\mathrm{C} 595$ and $\mathrm{Gd}^{3+}$ loading makes a novel nanobody with dual potential imaging and therapeutic effects on cancerous cells.

\section{Materials and Methods}

All chemical compounds and materials required for this study were provided by Sigma (USA) and used without any further purification. Cells were purchased from the Pasteur Institute of Iran. The experimental work was done in Shahid Beheshti University of Medical Sciences as well as an MR Imaging Center of Imam Khomeini Hospital, Tehran University of Medical Sciences during 2011-2012.

\subsection{Preparation of the $\mathrm{Gd}^{3+}-\mathrm{ALGDG}_{2}-\mathrm{C} 595$ Nano-Conjugate}

$\mathrm{ALGDG}_{2}$ was synthesized as follows: PEG-600 was chosen as the core and reacted with citric acid in the presence of excess amounts of thionyl chloride or dimethylaminopropyl carbodiimide (EDC) and the dialysis bag (cut off $2000 \mathrm{Da}$, Spectrum ${ }^{\circledR}$, USA) was used for the purification. To synthesize the C595-dendrimer conjugate, $75 \mu$ mole $\mathrm{ALGDG}_{2}$ was reacted with 0.01 mmole EDC and 0.05 mmole Sulfo-NHS in $2 \mathrm{ml}$ PBS or DDW for at least 5 minutes at $\mathrm{pH}$ of $5.5-6$ and the reaction allowed to reach to room temperature. Thereafter, activated dendrimer was added drop wise to the solution containing $1 \mu$ mole C595 in $2 \mathrm{ml}$ DDW medium in the presence of 1 mmole tri-ethyl-amine and the $\mathrm{pH}$ was adjusted to $7.5-8$ and the reaction allowed continuing for $12 \mathrm{hrs}$ at room temperature. To purify the conjugate the reaction mixture was dialyzed with cut off of $10 \mathrm{KDa}$ (Sigma, USA). To further purification the dialyzed solution was eluted through a Sephadex G-25 Fine ${ }^{\circledR}$ (Pharmacia-Fine Chemicals, Sweden) and the tubes containing the nano-conjugate were elected for the next step (e.g., lyophilization, $\mathrm{Gd}^{3+}$ loading). All steps were monitored by TLC (Thin Layer Chromatography) technique. Finally, $15 \mathrm{mmole} \mathrm{GdCl}_{3}$ was added to $1 \mu$ mole of nano-conjugate at room temperature and the reaction mixture was allowed to stir for at least $2 \mathrm{hrs}$ at $\mathrm{pH}$ of $7-7.5$. Afterward, to remove excess free $\mathrm{Gd}^{3+}$ ions the reaction $\mathrm{pH}$ was increased to 9 and free $\mathrm{Gd}^{3+}$ ions was precipitated and filtered. To increase the purity, the reaction mixture was dialyzed (Figures $\mathbf{1}$ and 2).

To purify dendrimer-antibody conjugate sephadex-G 25 fine $^{\circledR}$ (gel filtration chromatography) was used. Each external $\mathrm{ml}$ of eluent buffer of reaction mixture was collected separately in a numbered tube and its UV-OD at $280 \mathrm{~nm}$ was monitored to find antibody-dendrimer conjugate as well as non conjugated antibody. To find dendrimer conjugated C595 antibody ITLC (Iodine Thin Layer Chromatography) was performed.

High Pressure Liquid Chromatography (HPLC) was performed at small sample amounts using either $10 \mathrm{~mm} \times$ $250 \mathrm{~mm}$ pre-packed column at room temperature.

\subsection{Gd $^{3+}$-FT-IR Spectroscopy}

To investigate whether or not the changes occurred after $\mathrm{Gd}^{3+}$ loading FT-IR spectroscopy was employed by performance on nano-conjugate before and after $\mathrm{Gd}^{3+}$ loading.

\subsection{Total Protein Measurement}

The assay performed in microplates based on micro Lowry [16-18] that has been adapted to microplates. The reagents were provided from (Catalogue No. 690-A: Sigma Chemical, St. Louis MO).

A standard curve was prepared as follow: Bvine serum albumin (BSA) powder was dissolved in distilled water and diluted to a concentration of $1 \mu \mathrm{g} / \mu \mathrm{l}$. A series of dilutions $(5,10,20,40,60,80$, and $100 \mu \mathrm{g} /$ well) were prepared and used in tetraplicate/100 $\mu$ l. Different concentarions of protein were prepared $(0-100 \mu \mathrm{g} / 100 \mu \mathrm{l})$ and a standard curve was obtained. The same procedure was performed for $\mathrm{Gd}^{3+}$ loaded nano-conjugate and C595 alone and based on the comparisons and standard curve diagram and equation unknown sample was determined.

\subsection{Size Measurement and SEM/TEM Images}

Nanosize was determined using a Brookhaven ZetaPALS 


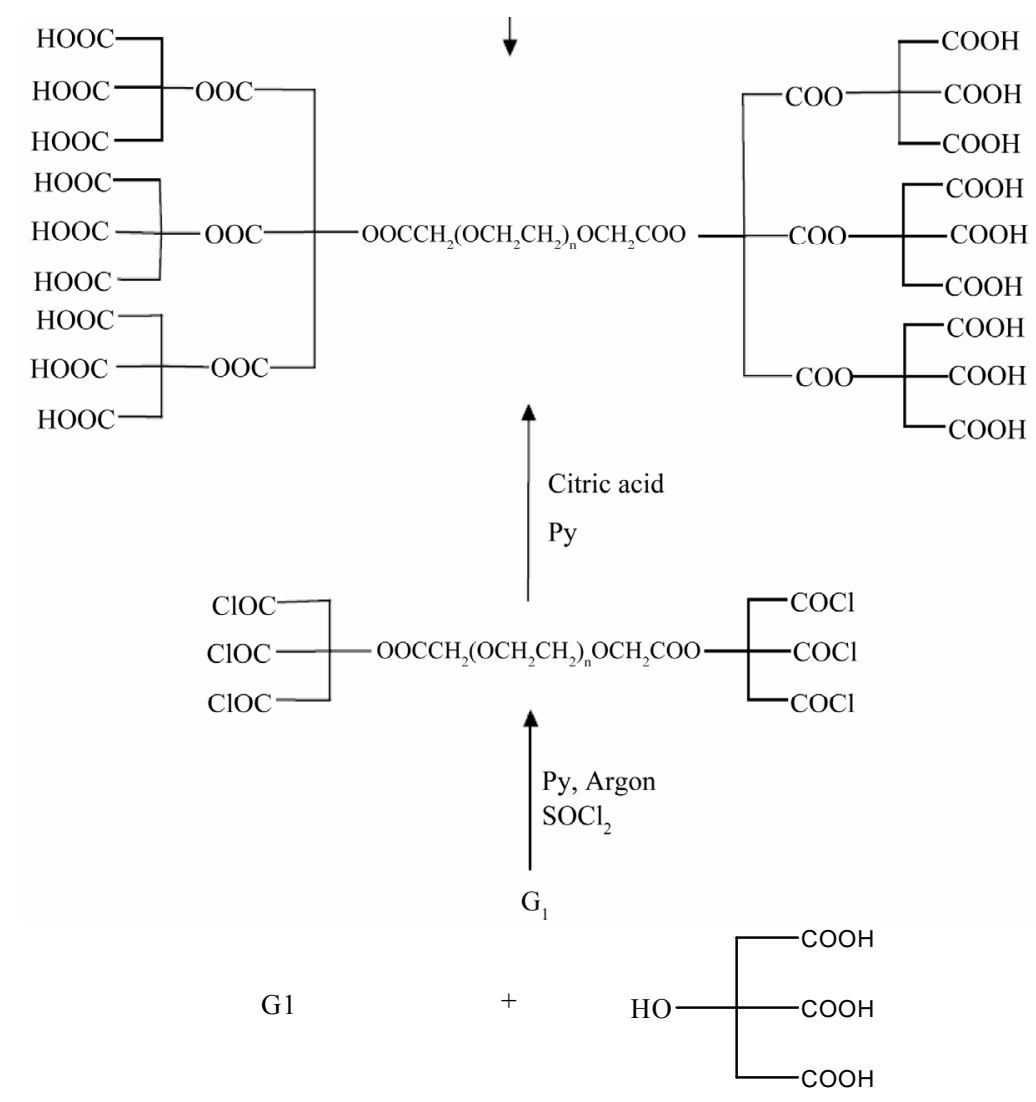

$$
\begin{array}{l|l}
\text { r.t } & \begin{array}{l}
\mathrm{DCC} \\
\mathrm{Py}
\end{array}
\end{array}
$$

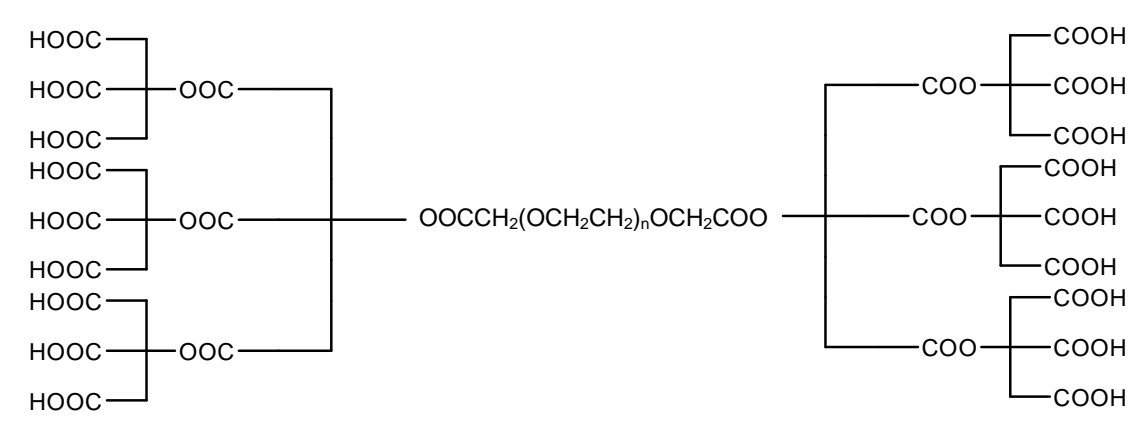

Figure 1. Schematic of chemical synthesis pathway of ALGDG .

system. Beckman Multisizer Coulter equipped with a 1 $\mathrm{nm}$ or $100 \mathrm{~nm}$ apertures has been performed to depict the particle size and zeta potential distribution. The conjugates were also characterized by Transmission Electron Microscopy (JOEL 1230; accelerating voltage, $100 \mathrm{kV}$ ). For TEM of samples, one drop of $\mathrm{Gd}^{3+}-\mathrm{ALGDG}_{2}-\mathrm{C} 595$ aqueous suspension was dispersed on copper grid and the excess volume was removed. The samples were used after drying at room temperature.

\subsection{Relaxivity Measurement}

The logarithmic water proton relaxivity ratio at different lamor frequencies $(\mathrm{MHz})$ was determined by using a 


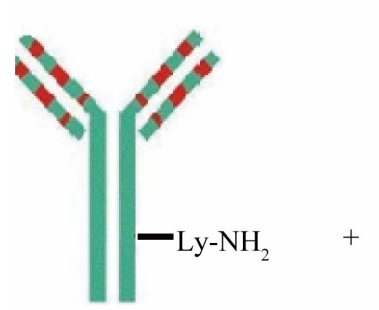

C595

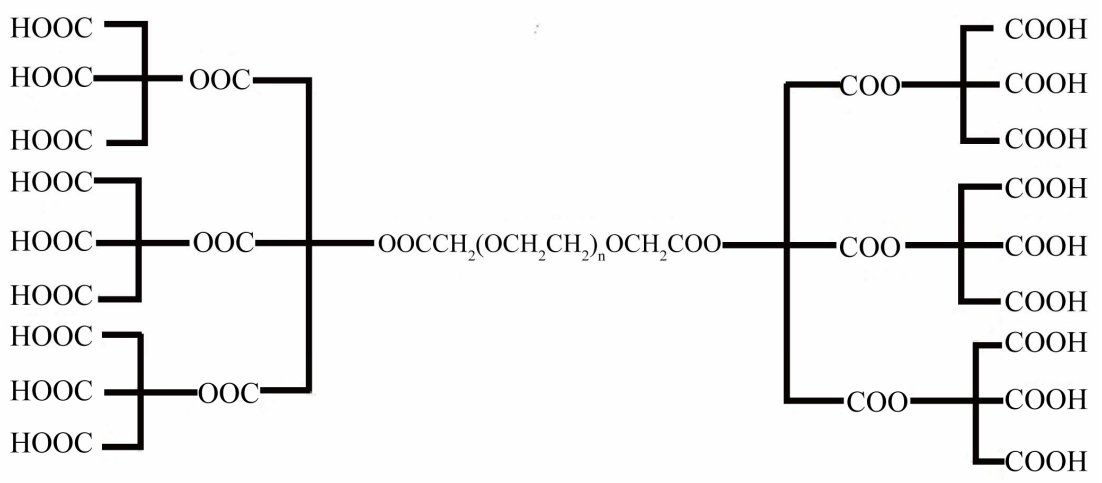

G2

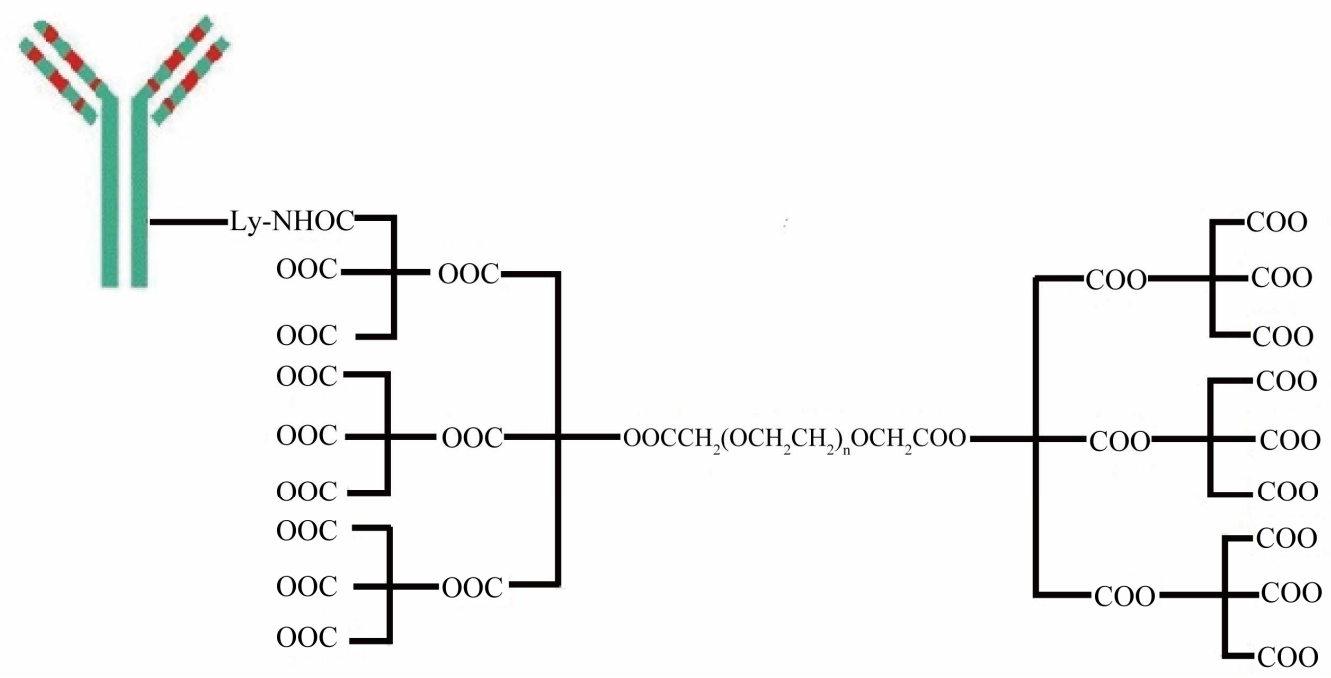

Figure 2. Synthesis pathway of $\mathrm{ALGDG}_{2}$, its $\mathrm{Gd}^{3+}$ loading and conjugation with $\mathbf{C 5 9 5}$.

Spinmaster spectrometer (Stelar, Mede, Italy) set at $0.5 \mathrm{~T}$; the inversion recovery method was used (number of experiments 16 and 4 scans). $90^{\circ}$-pulse width was set 3.5 milliseconds (ms), and the reproducibility error of the $\mathrm{T}_{1}$ data was $\pm 0.75 \%$. The temperature was monitored by an air-flow heater equipped with a copper-constantan thermocouple. The proton $1 / \mathrm{T}_{1}$ NMRD profiles were measured on a Koenig-Brown field-cycling relaxometer over a continuum of magnetic field strengths from 0.00024 to $1.2 \mathrm{~T}$. More explorations about data calculation procedure was reported previously [19].

\subsection{Stability}

The relaxation measurements were performed as same as the relaxivity studies but in the presence of $20 \%$ solution of EDTA (as $\mathrm{Gd}^{3+}$ chelator) for at least $24 \mathrm{hrs}$ according to the protocol described previously [20].

\subsection{Statistical Analysis}

All the experiments were conducted in triplicate $(n=3)$ and were compared using one-way ANOVA for mean comparison of more than two samples and post-hoc experiments (multiple comparisons) with Dunnett test with SPSS-16 software. The results were reported as significant for $P<0.05$ or highly significant for $P<0.01$.

\section{Results}

\subsection{Purification Assay}

As shown in Figure 3(a) tube numbers 13 - 16 contained $\mathrm{ALGDG}_{2}-\mathrm{C} 595$ and this confirmed by TLC. Its retention factor (Rf) was completely differed from dendrimer alone and $\mathrm{C} 595$ or other reaction reagents. Further identifications performed by RP-HPLC, the data shown a retention time of 7, 12 and 13 min for dendrimer, C595 


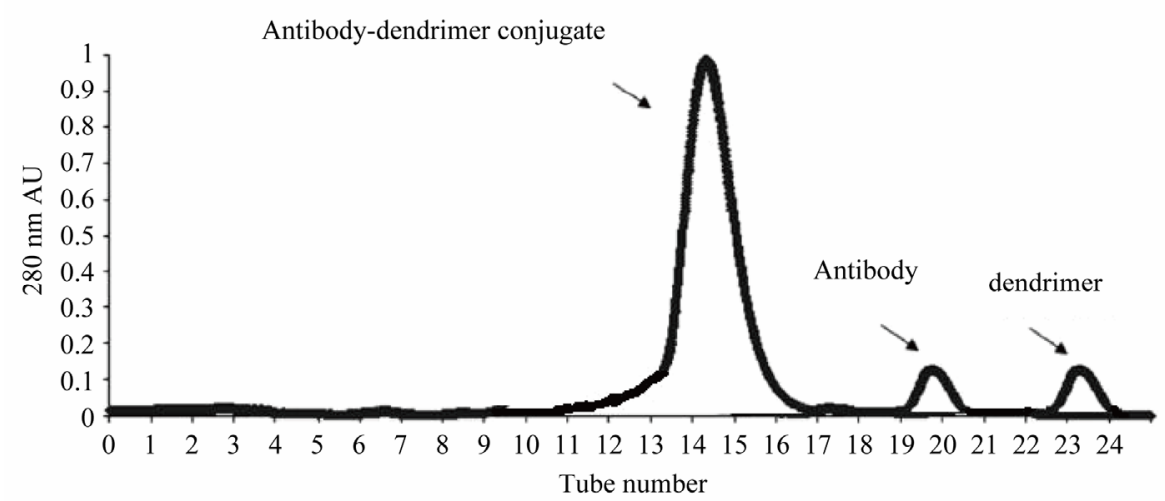

(a)

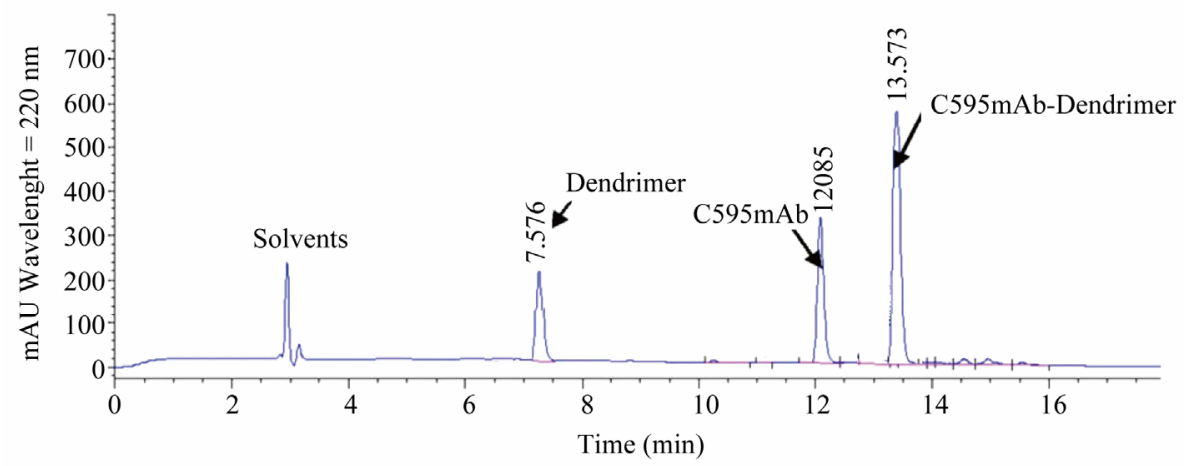

(b)

Figure 3. (a) Schematic illustration of nano-conjugate dendrimer-C595 purification and (b) Analytical RP-HPLC chromatogram.

and dendrimer-C595 conjugate, respectively. As can be seen from Figure 3(b), the purity of the compounds was obtained more than $95 \%$ according to AUC, area under the curve of each peak.

\subsection{FT-IR Spectroscopy}

Clear changes in FTIR peaks before and after $\mathrm{Gd}^{3+}$ loading was occurred and this indicates the essential interaction of $\mathrm{Gd}^{3+}$ ions and dendrimer-C595 conjugate (Figure 4).

\subsection{Protein Assay}

Each $100 \mu \mathrm{g}$ nanoconjugate contained $84.3 \pm 4 \mu \mathrm{g}$ protein regarded to Mab C595 while each $100 \mu \mathrm{g}$ intact Mab C595 contained $>98 \mu \mathrm{g}$ protein (Figure 5).

\section{4. $\mathrm{Gd}^{3+}$ Loading and Size SEM/TEM Results}

Inductively Coupled Plasma Mass data showed $11.01 \pm$ $4 \%(\mathrm{n}=3) \mathrm{Gd}^{3+}$ in each $100 \mu \mathrm{g}$ nanoconjugate. Size distribution showed that dendrimer alone has an average size of $25 \mathrm{~nm}$ and after conjugation and $\mathrm{Gd}^{3+}$ loading the average aggregation size reached to $61 \mathrm{~nm}$ (Figure 6).
The apparent views of nanoparticles were also shown in SEM and TEM images. The cryo TEM of $\mathrm{Gd}^{3+}$-dendrimer-C595 showed high average more than $2 \mathrm{~nm}$ (Figures $7(a)-(d))$.

\subsection{Relaxivity Studies}

The relaxivity data were illustrated in Figure 8. As it can be found $\mathrm{Gd}^{3+}$ loaded nanoconjugate showed a concentration intendancy. Increases in concentration of nonoconjugate caused increases in the paramagnetic potential of the nano-probe and leads to its application as a MR imaging probe.

\section{Discussion}

The present study showed a successful strategy in generating a powerful nanobiomolecular probe considering the biocompatibility and appropriate $\mathrm{Gd}^{3+}$ loading capability to find and treat breast cancer with enough relaxivity. The first claim for the study is to synthesis the $\mathrm{ALGDG}_{2}$ and to conjugate with Mab C595 (anti-MUC1 breast and bladder cancer receptor) in a manner of not producing any instability/inconsistency in the antibody pharma- 


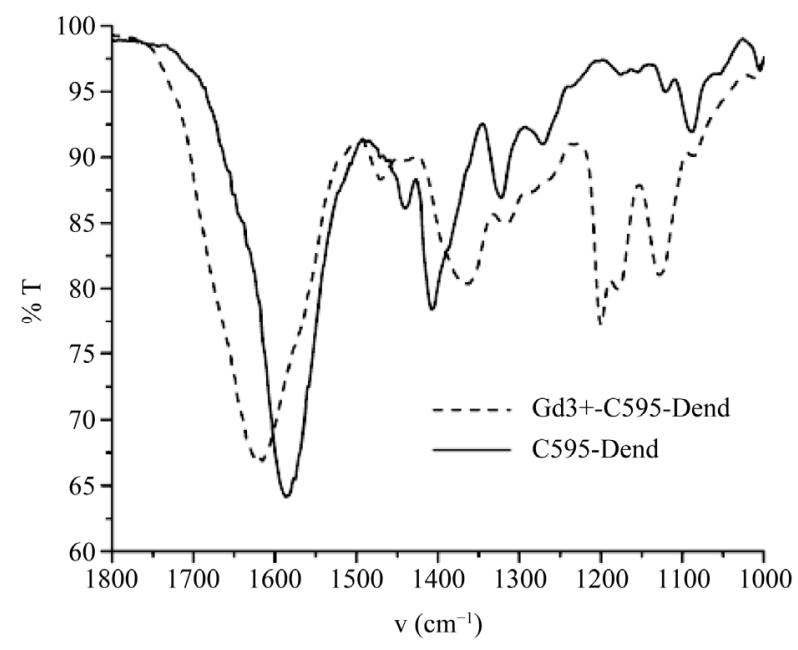

Figure 4. Changes occurred before and after $\mathbf{G d}^{3+}$ loading on ALGDG $_{2}$-C595 nanoconjugate.

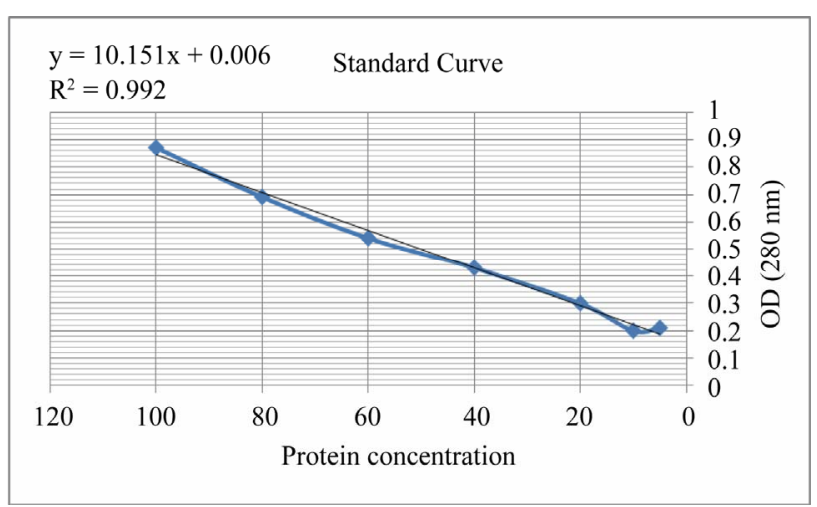

Figure 5. Illustration of the obtained standard curve used for protein content assay.
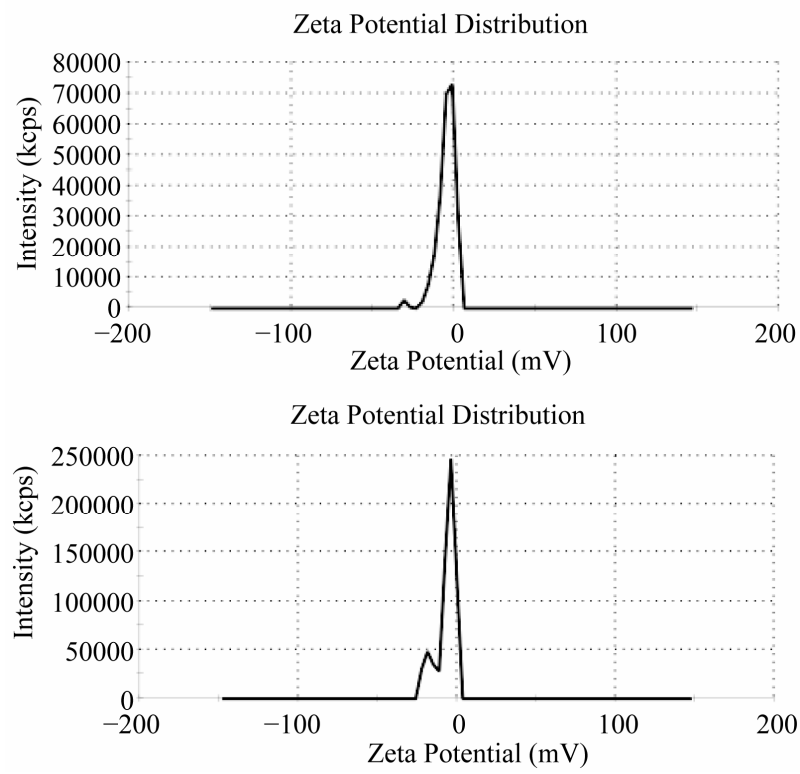

Figure 6. Illustration of size distribution of dendrimer before and after $\mathrm{Gd}^{3+}$ and $\mathrm{C} 595$ bioconjugation. cokinetics and dynamics (Figure 3(b)).

While the first claim was strictly found right, the second claim was to investigate the capability of bionanocontrast agent to produce enough relaxivity $\left({ }^{1} \mathrm{H}-\mathrm{NMRD}\right)$ and specific binding to MUC-1 receptor expressing cells as well as anti-breast cancer cell activity in vitro. Finally, $\mathrm{Gd}^{3+}$ loaded nanoconjugate were found quite successful in the all biological experiments performed (Figures 4 and $\mathbf{8}$ ).

The advantageous of $\mathrm{ALGDG}_{2}$ to PAMAM (Polyamidoamine) dendrimer is describing as follows; first, PAMAM needs to be re-functionalized with a $\mathrm{Gd}^{3+}$ chelator such diethylenetriaminepentaacetic acid (DTPA) in a very difficult manner and second, PAMAM did not show enough water solubility as well as safety. Third advantage for the $\mathrm{ALGDG}_{2}$ is regarding to its PEG core which makes it mostly attractive to cancerous cells as well as anticancer effects in a good agreement with findings of the other works $[18,19,21,22]$. The next interesting capability of $\mathrm{ALGDG}_{2}$ is regarding to its citric acid shell which raise potent $\mathrm{Gd}^{3+}$ complex formation as well as $\mathrm{Gd}^{3+}$ loading. The negative charge of dendrimer $\mathrm{G}_{2}$ protect nanoprobe from any surface-surface toxic interactions between the normal cell body and the conjugate. DTPA is as an extrcellular MR imaging contrast agent due to its reduced uptake by cells and this is a disadvantage of the compound [15]. To increase the cellular uptakes, in particular, in cancer cell a well recognized dendrimer conjugation was applied here.

One of the main characteristics of biomolecular conjugation is regarding to not producing any biological activity complications. The complications occur while one or both biomolecule's active site (covering kinetic or dynamic active site) suffers an inactivation and this is sometimes rationally and this is in agreement with other reported results [17-19,21-23].

As a result and based on the obtained in vitro observations, Mab C595 serves its activity after dendrimer conjugation and this fact leads to next nano-conjugate in vivo success. In confirmation, there are some reports on the anticancer activity of unbound $\mathrm{C} 595$ which suggests anticancer effects for C595 [19,21,22].

Low doses of the conjugate showed a less paramagnetic activity but, it is interesting that by increasing the concentrations of nonoconjugate, the relaxation time is also increased and leads to using of this nonoprobe as an MR imaging contrast agents.

\section{Conclusion}

Based on the confirmations from the present research $\mathrm{Gd}^{3+}-\mathrm{ALGDG}_{2}-\mathrm{C} 595$ nano-probe which synthsised and examined for the first time in this study, may be a potential dual selective breast molecular imaging and seems to 


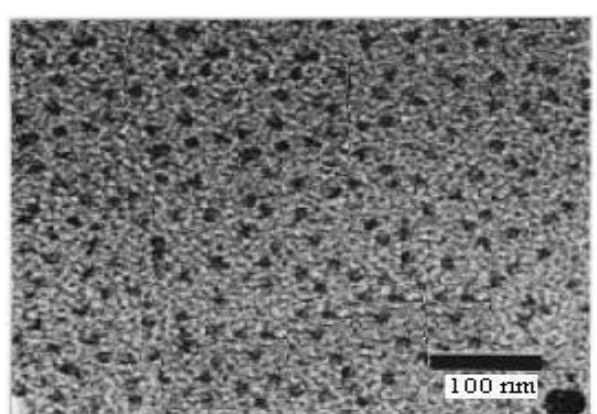

(a)

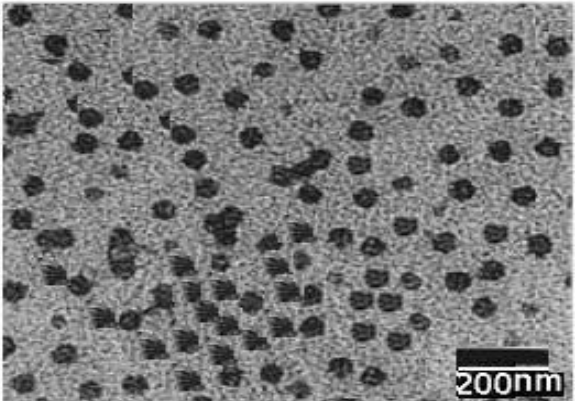

(b)

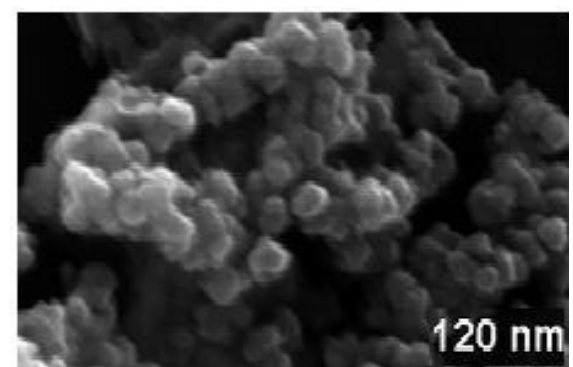

(c)

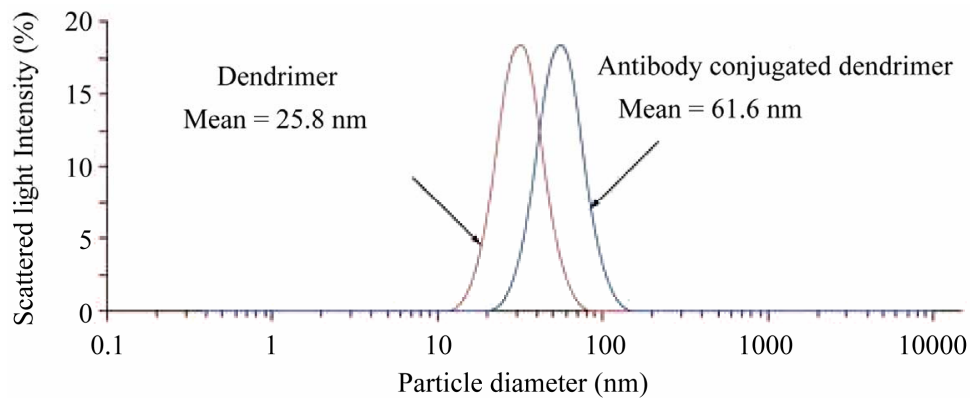

(d)

Figure 7. (a) Illustration of dendrimer TEM; (b) Gd-dendrimer-C595; (c) Gd-dendrimer-C595 (SEM) and (d) Size distribution of dendrimer before and after $\mathrm{Gd}^{3+}$ and $\mathrm{C} 595$ bioconjugation.

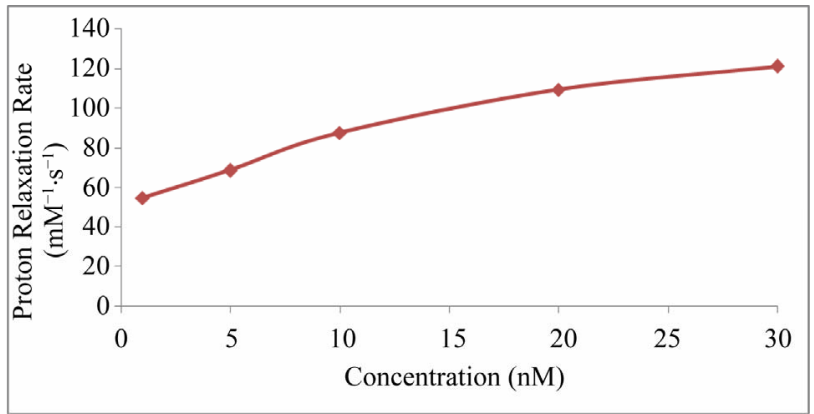

Figure 8. Curve of proton relaxation rate $\left(\mathrm{mM}^{-1} \cdot \mathrm{s}^{-1}\right)$ versus different concentration of nanoconjugate (nM).

be a functional nano-probe in the near future and this needs to be further investigated by in vivo experiments and clinical trials.

\section{Acknowledgements}

This research is a major part of a Ph.D. thesis in Nan- medicine by Dr. Mehdi Mirzaei. This study was supported by Shahid Beheshti University of Medical Sciences as well as MR Imaging Center of Imam Khomeini Hospital, Tehran University of Medical Sciences, Iran. The authors wish to thank all technicians who provided support during the course of research.

\section{REFERENCES}

[1] A. L. Baert and K. Sartor, "Medical Radiology Diagnostic Imaging," Springer, Heidelberg, 2006.

[2] S. A. Eccles, G. Box, W. Court, J. Sandle and C. J. Dean, "Preclinical Models for the Evaluation of Targeted Therapies of Metastatic Disease," Cell Biophysics, Vol. 24-25, No. 1-3, 1994, pp. 279-291.

[3] K. N. Raymond and V. C. Pierre, "Next Generation, High Relaxivity Gadolinium MRI Agents," Bioconjugate Chemistry, Vol. 16, No. 1, 2005, pp. 3-8. doi:10.1021/bc049817y

[4] C. J. Mettlin, G. P. Murphy, R. Ho and H. R. Menck, 
"The National Cancer Data Base Report on Longitudinal Observations on Prostate Cancer," Cancer, Vol. 77, No. 10, 1996, pp. 2162-2166. doi:10.1002/(SICI)1097-0142(19960515)77:10<2162::AI D-CNCR30>3.0.CO;2-R

[5] C. Galasko, "The Anatomy and Pathways of Skeletal Metastases," In: L. Weiss and A. H. Gilbert, Eds., Bone Metastases, G. K. Hall, Boston, 1981, pp. 49-63.

[6] M. S. O'Reilly, L. Holmgren, Y. Shing, C. Chen, R. A. Rosenthal, M. Moses, W. S. Lane, Y. Cao, E. H. Sage and J. Folkman, "Angiostatin: A Novel Angiogenesis Inhibitor That Mediates the Suppression of Metastases by a Lewis Lung Carcinoma," Cell, Vol. 79, No. 2, 1994, pp. 315-328. doi:10.1016/0092-8674(94)90200-3

[7] L. Wang, H. Chen, M. H. Pourgholami, J. Beretov, J. Hao, H. Chao, A. C. Perkins, J. H. Kearsley and L. Yong, "AntiMUC1 Monoclonal Antibody (C595) and Docetaxel Markedly Reduce Tumor Burden and Ascites and Prolong Survival in an in Vivo Ovarian Cancer Model," Plos One, Vol. 6, No. 9, 2011, e24405.

doi:10.1371/journal.pone.0024405

[8] D. J. Yang, C. G. Kim, N. R. Schechter, A. Azhdarinia, D. F. Yu, C. S. Oh, J. L. Bryant, E. E. Kim and D. A. Podoloff, "Imaging with ${ }^{99} \mathrm{Tc}^{\mathrm{m}}$-ECDG Targeted at the Multifunctional Glucose Transport System: Feasibility Study with Rodents," Radiology, Vol. 226, 2003, pp. 465-473. doi:10.1148/radiol.2262011811

[9] C. F. Geraldes and S. Laurent, "Classification and Basic Properties of Contrast Agents for Magnetic Resonance Imaging", Contrast Media and Molecular Imaging, Vol. 4, No. 1, 2009, pp. 1-23. doi:10.1002/cmmi.265

[10] I. Haririan, M. S. Alavidjeh, M. R. Khorramizadeh, M. S. Ardestani, Z. Z. Ghane and H. Namazi, "Anionic LinearGlobular Dendrimer-Cis-Platinum (II) Conjugates Promote Cytotoxicity in Vitro against Different Cancer Cell Lines," International Journal of Nanomedicine, Vol. 5, 2010, pp. 63-75. doi:10.2147/IJN.S8595

[11] M. S. Alavidjeh, I. Haririan, M. R. Khorramizadeh, Z. Z. Ghane, M. S. Ardestani and H. Namazi, "Anionic LinearGlobular Dendrimers: Biocompatible Hybrid Materials with Potential Uses in Nanomedicine," Jopurnal of Material Sciences and Material Medicine, Vol. 21, No. 4, 2010, pp. 1121-1133. doi:10.1007/s10856-009-3978-8

[12] M. Amanlou, S. D. Siadat, S. E. S. Ebrahimi, A. Alavi, M. R. Aghasadeghi, M. S Ardestani, S. Shanehsaz, M. Ghorbani, B. Mehravi, M. S. Alavidjeh, A. Jabbari-Arabzadeh and M. Abbasi, "Gd ${ }^{3+}$-DTPA-DG: Novel Nanosized Dual Anticancer and Molecular Imaging Agent," International Journal of Nanomedicine, Vol. 6, 2011, pp. 747-763. doi:10.2147/IJN.S17648

[13] D. Shahbazi-Gahrouei, "Novel MR Imaging Contrast Agents for Cancer Detection," Journal of Research in Medical Sciences, Vol. 14, No. 3, 2009, pp. 141-147.
[14] R. Singh and D. Bandyopadhyay, "MUC-1: A Target Molecule for Cancer Therapy," Cancer Biology and Therapy, Vol. 6, No. 4, 2007, pp. 481-486. doi:10.4161/cbt.6.4.4201

[15] D. Shahbazi-Gahrouei, M. Williams, S. Rizvi and B. J. Allen, "In Vivo Studies of Gd-DTPA-Monoclonal Antibody and Gd-Porphyrins: Potential MR Imaging Contrast Agents for Cancer," Journal of Magnetic Resonance Imaging, Vol. 14, No. 2, 2001, pp. 169-174. doi:10.1002/jmri.1168

[16] S. M. Cohen, J. D. Xu, E. Radkov, K. N. Raymond, M. Botta, A. Barge and S. Aime, "Syntheses and Relaxation Properties of Mixed Gadolinium Hydroxypyridinonate MRI Contrast Agents," Inorganic Chemistry, Vol. 39, No. 25, 2000, pp. 5747-5756. doi:10.1021/ic000563b

[17] S. H. Koenig and R. D. Brown, "NMR Spectroscopy of Cells and Organism," CRC Press, Boca Raton, 1987, p. 756.

[18] S. Von Mensdorff-Pouilly, M. M. Gouretvich, P. Kenemans, A. A. Verstraeten, G. J. Van Kamp, et al., "An Enzyme-Linked Immunosorbent Assay for the Measurement of Circulating Antibodies to Polymorphic Epithelial Mucin (MUC-1)," Tumor Biology, Vol. 19, No. 3, 1998, pp. 186-195. doi: $10.1159 / 000030006$

[19] G. Parnaud, S. Tache, G. Peiffer and D. E. Corpet, "Polyethylene-Glycol Suppresses Colon Cancer and Causes Dose-Dependent Regression of Azoxymethane-Induced Aberrant Crypt Foci in Rats," Cancer Research, Vol. 59, No. 20, 1999, pp. 5143-5147.

[20] M. Mirzaei, M. A. Mohagheghi, D. Shahbazi-Gahrouei and A. Khatami, "Novel Nanosized Gd $^{3+}-\mathrm{ALGD}_{-} \mathrm{G}_{2}$ C595: In Vivo Dual Selective MUC-1 Positive Tumor Molecular MR Imaging and Therapeutic Agent," Journal of Nanomedicine and Nanotechnology, Vol. 3, No. 147, 2012, Article ID: 1000147. doi:10.4172/2157-7439.1000147

[21] S. Giray, T. Bal, A. M. Kartal, S. Kizılel and C. Erkey, "Controlled Drug Delivery through a Novel PEG Hydrogel Encapsulated Silica Aerogel System," Journal of Biomedical Materials Research A, Vol. 100, No. 5, 2012, pp. 1307-1315. doi:10.1002/jbm.a.34056

[22] N. Fischer-Durand, M. Salmain, B. Rudolf, L. Dai, L. Juge, V. Guérineau, O. Laprévote, A. Vessières and G. Jaouen, "Site-Specific Conjugation of Metal Carbonyl Dendrimer to Antibody and Its Use as Detection Reagent in Immunoassay," Analytical Biochemistry, Vol. 407, No. 2, 2010, pp. 211-219. doi:10.1016/j.ab.2010.08.027

[23] P. K. Smith, R. I. Krohn, G. T. Hermanson, A. K. Mallia, F. H. Gartner, M. D. Provenzano, E. K. Fujimoto, N. M. Goeke, B. J. Olson and D. C. Klenk, "Measurement of Protein Using Bicinchoninic Acid," Analytical Biochemistry, Vol. 150, No. 1, 1985, pp. 76-85. doi:10.1016/0003-2697(85)90442-7 